\title{
Meson mass spectrum at finite temperature and density in the strong coupling limit of lattice QCD for color SU(3)
}

\author{
Noboru Kawamoto, Kohtaroh Miura* and Akira Ohnishi \\ Hokkaido University \\ E-mail: miura@particle.sci.hokudai.ac.jp
}

We investigate the meson mass spectrum in the strong coupling limit of lattice QCD with one species of staggered fermion for the $\mathrm{SU}\left(N_{c}\right)$ color gauge group, including $N_{c}=3$. We analytically derive meson masses as functions of temperature and chemical potential via chiral condensates. We show that meson masses quickly decrease to zero when the chemical potential or the temperature approaches to the critical value.

The XXV International Symposium on Lattice Field Theory

July 30-4 August 2007

Regensburg, Germany

\footnotetext{
* Speaker.
} 


\section{Introduction}

It is a common folklore that the chiral phase transition takes place at high temperature and/or density in Quantum Chromodynamics (QCD). Meson masses are crucially influenced by the chiral condensate, and thus are very interesting observables in the chiral phase transition region.

It is possible to extract hadron masses quantitatively in the lattice Monte-Carlo (MC) simulations at zero density. However, MC does not work well at high densities because of the notorious negative sign problem of the Dirac determinant. Therefore it is instructive to investigate high density matter in the strong coupling lattice QCD (SC-LQCD). In SC-LQCD analyses with a mean field approximation (MFA), we can derive analytical expressions of the effective potential as a function of temperature $(T)$ and chemical potential $(\mu)$, and hence we can avoid the sign problem. The numerical values of physical observables derived analytically in SC-LQCD should be reproduced in lattice MC simulations at least in the strong coupling region. From this point of view, Refs. [1] provides an interesting comparison of strong coupling results in $\mathrm{MC}$ and analytic studies.

Chiral phase transitions are governed by the effective potential, which also plays an essential role to obtain the hadron mass in SC-LQCD. The effective potential at finite temperature and density has been extensively studied under various conditions [2-7]. For example, we have recently derived analytical expressions of the effective potential as a function of $T$ and $\mu$ in SC-LQCD, including the baryon propagating effects or $1 / g^{2}$ corrections [7]. On the other hand, while hadron masses have been studied at zero temperature [6, 8, 9], the expression of meson masses as a function of $T$ and $\mu$ has never been derived before.

In this proceedings, we derive an analytical expression of meson masses as functions of the chiral condensate, which is a function of $T$ and $\mu$ in SC-LQCD for color $\mathrm{SU}\left(N_{c}\right)$.

\section{A brief summary of meson mass derivation}

We start from the action and partition function of lattice QCD with one species of staggered fermion $(\chi)$ in the strong coupling limit, where we omit the pure gauge plaquette terms $\left(\propto 1 / g^{2}\right)$,

$$
\begin{aligned}
Z & \left.=\int_{\chi, \bar{\chi}, U_{0}, U_{j}} \exp \left[-\frac{1}{2} \sum_{x} \sum_{j=1}^{d}(-1)^{x_{0}+\cdots+x_{j}}\left[\bar{\chi}_{x} U_{j}(x) \chi_{x+\hat{j}}-(h . c)\right]-S_{F}^{t}-m_{0} \sum_{x} M_{x}\right)\right] \\
S_{F}^{t} & =\frac{1}{2} \sum_{x}\left[e^{\mu} \bar{\chi}_{x} U_{0}(x) \chi_{x+\hat{0}}-e^{-\mu}(h . c)\right], \quad M_{x}=\sum_{a=1}^{N_{c}}\left(\bar{\chi}^{a} \chi_{a}\right)_{x} .
\end{aligned}
$$

Here $m_{0}, U_{0}, U_{j}$ represent the current quark mass, the temporal and spatial link variables, respectively. We introduce the lattice chemical potential $\mu$ following the procedure in Ref. [10].

First we perform the path integral over spatial link variables $U_{j}$, and keep only the leading order terms in the $1 / d$ expansion,

$$
Z=\int_{\chi, \bar{\chi}, U_{0}} \exp \left[-S_{F}^{t}+\frac{\sum_{x, j} M_{x} M_{x+\hat{j}}}{4 N_{c}}-m_{0} \sum_{x} M_{x}+\mathscr{O}(1 / \sqrt{d})\right] .
$$


Next we introduce the chiral condensate $\sigma \propto\langle\chi \bar{\chi}\rangle$ through the bosonization procedure,

$$
\begin{aligned}
Z & \simeq \int_{\chi, \bar{\chi}, U_{0}, \sigma} \exp \left[-\frac{1}{2} \sum_{m n, \mathbf{x y}} \sigma_{m \mathbf{x}} V_{M}^{-1}(\mathbf{x y}) \delta_{m n} \sigma_{n \mathbf{y}}-\sum_{n \mathbf{x}} m_{n \mathbf{x}}^{(q)} M_{n \mathbf{x}}-S_{F}^{t}\right], \\
V_{M}(\mathbf{x y}) & =\frac{1}{4 N_{c}} \sum_{j=1}^{d}\left(\delta_{\mathbf{x}+\hat{j}, \mathbf{y}}+\delta_{\mathbf{x}-\hat{j}, \mathbf{y}}\right), \quad m_{n \mathbf{x}}^{(q)}=m_{0}+\sigma_{n \mathbf{x}},
\end{aligned}
$$

where " $m$ " or " $n$ " denotes the temporal lattice site, which takes an integer value in $[1, N]$. Now we can perform the path integral over staggered quarks, and obtain the following effective action of $\sigma$ up to a constant,

$$
Z \simeq \int \mathscr{D} \boldsymbol{\sigma} e^{-S_{\mathrm{eff}}[\boldsymbol{\sigma}]}, \quad S_{\mathrm{eff}}[\boldsymbol{\sigma}]=\frac{1}{2} \sum_{m n, \mathbf{x y}} \sigma_{m \mathbf{x}} V_{M}^{-1}(\mathbf{x y}) \boldsymbol{\delta}_{m n} \sigma_{n \mathbf{y}}-\sum_{\mathbf{x}} \log R_{\mathbf{x}}[\boldsymbol{\sigma}]
$$

Integral over $\chi$ and $U_{0}$ generates the interaction term $\log R_{\mathbf{x}}[\sigma]$, where $R_{\mathbf{x}}[\sigma]$ is given as,

$$
R_{\mathbf{x}}[\boldsymbol{\sigma}]=\int d U_{0}(\mathbf{x})\left|\begin{array}{ccccc}
2 m_{1, \mathbf{x}}^{(q)} \mathbf{1}_{c} & e^{\mu} \mathbf{1}_{c} & & & e^{-\mu} U_{0}^{\dagger}(\mathbf{x}) \\
-e^{-\mu} \mathbf{1}_{c} & 2 m_{2, \mathbf{x}}^{(q)} \mathbf{1}_{c} & e^{\mu} \mathbf{1}_{c} & \mathbf{0} & \\
& \ddots & \ddots & \ddots & \\
& \mathbf{0} & -e^{-\mu} \mathbf{1}_{c} & 2 m_{N-1, \mathbf{x}}^{(q)} \mathbf{1}_{c} & e^{\mu} \mathbf{1}_{c} \\
-e^{\mu} U_{0}(\mathbf{x}) & & & -e^{-\mu} \mathbf{1}_{c} & 2 m_{N, \mathbf{x}}^{(q)} \mathbf{1}_{c}
\end{array}\right|
$$

where $\mathbf{1}_{c}$ represents an $N_{c} \times N_{c}$ unit matrix, and $U_{0}$ is given in the temporal gauge as,

$$
U_{0}(\mathbf{x})=\operatorname{diag}\left\{e^{i \theta^{1}(\mathbf{x})}, \cdots, e^{i \theta^{N_{c}}(\mathbf{x})}\right\} .
$$

Periodic and anti-periodic boundary conditions for gluons and fermions are respected in the present finite $T$ treatment as found in the form of Eq. (2.7), where we find different signs in the upper-right and the lower-left components.

We decompose the chiral condensates $\sigma_{n}(\mathbf{x})$ to the equilibrium value $\bar{\sigma}$ and fluctuations $\delta \sigma_{n}(\mathbf{x})$, $\sigma_{n}(\mathbf{x})=\bar{\sigma}+\delta \sigma_{n}(\mathbf{x})$. The equilibrium value $\bar{\sigma}$ is determined from the stationary condition of the effective action,

$$
\left.\frac{\delta S_{\mathrm{eff}}[\boldsymbol{\sigma}]}{\delta \sigma_{n \mathbf{x}}}\right|_{\sigma \rightarrow \bar{\sigma}}=\bar{\sigma} \frac{2 N_{c}}{d}-\left.\frac{1}{R[\overline{\boldsymbol{\sigma}}]} \frac{\delta R_{\mathbf{x}}[\sigma]}{\delta \sigma_{n \mathbf{x}}}\right|_{\sigma \rightarrow \overline{\boldsymbol{\sigma}}}=0 .
$$

The meson mass is defined as the pole of the propagator for $\delta \sigma$, which is obtained from the second order variation of the effective action $\left(\delta_{\sigma}^{2} S_{\text {eff }}\right)$. The stationary condition Eq. (2.9) ensures that the first derivative of $R_{\mathbf{x}}[\sigma]$ is independent from the space-time point. Thus when we require the null average fluctuation condition $\sum_{n} \delta \sigma_{n}=0$, the first derivative of $R_{\mathbf{x}}[\sigma]$ in $\delta_{\sigma}^{2} S_{\text {eff }}$ disappears. The second order variation $\delta_{\sigma}^{2} S_{\text {eff }}$ is found to be,

$$
\left.\delta_{\sigma}^{2} S_{\mathrm{eff}}[\boldsymbol{\sigma}]\right|_{\sigma \rightarrow \bar{\sigma}}=\sum_{m n, \mathbf{x y}} \delta \sigma_{m \mathbf{x}}\left[V_{M}^{-1}(\mathbf{x y}) \delta_{m n}-\left.\delta_{\mathbf{x y}} \frac{R_{m n}^{(2)}[\boldsymbol{\sigma}]}{R[\boldsymbol{\sigma}]}\right|_{\sigma \rightarrow \bar{\sigma}}\right] \delta \sigma_{n \mathbf{y}}, \quad R_{m n}^{(2)} \equiv \frac{\delta^{2} R_{\mathbf{x}}}{\delta \sigma_{m} \delta \sigma_{n}}
$$

It is necessary to evaluate $R_{m n}^{(2)}$ to obtain meson masses. We derive $R_{m n}^{(2)}$ at finite $T$ and $\mu$ in the following section, which has not been derived previously. 


\section{Evaluation of quark hopping in the temporal direction}

We utilize the formulation developed by Damgaard, Kawamoto, Shigemoto [2], Faldt and Petersson [3], and Nishida [4]. First we reduce the $\left(N_{c} \times N\right) \times\left(N_{c} \times N\right)$ determinant in Eq. (2.7) to $N_{c} \times N_{c}$ determinant in the form [3],

$$
R_{\mathbf{x}}=\int d U_{0} \operatorname{det}_{c}\left[X_{N} \otimes \mathbf{1}_{c}+\left(e^{\mu / T} U_{0}+e^{-\mu / T} U_{0}^{\dagger}\right)\right]=R_{\mathbf{x}}\left(X_{N}[\boldsymbol{\sigma}], \mu\right),
$$

where the quark hopping kernel $X_{N}$ is a functional of $\sigma$. We assume that the number of temporal lattice sites $N$ is even and its inverse $N^{-1}$ is identified as the temperature $T$. Since $R_{\mathbf{x}}$ is a function of $X_{N}$, it is enough to evaluate the $U_{0}$ integral in equilibrium, and this has been done by utilizing the Vandermonde determinant technique combined with the recursion formula [2, 4]. It is also possible to perform the $U_{0}$ integral explicitly by using the one link integral technique [3]. The obtained $R_{\mathbf{x}}$ reads,

$$
R_{\mathbf{x}}\left(X_{N}[\sigma]=Y+Y^{-1}, \mu\right)=\frac{Y^{N_{c}+1}-Y^{-\left(N_{c}+1\right)}}{Y-Y^{-1}}+2 \cosh \frac{N_{c} \mu}{T} .
$$

In this proceedings, the baryonic effects are considered for just a temporal direction, which is reflected on the second term in Eq (3.2).

Next it is necessary to evaluate the quark hopping kernel $X_{N}$, which is found to be [3],

$$
X_{N}=B_{1, \cdots, N}+B_{2, \cdots, N-1}, \quad B_{1, \cdots, N}=\left|\begin{array}{ccccc}
2 m_{1, \mathbf{x}}^{(q)} & e^{\mu} & & & \\
-e^{-\mu} & 2 m_{2, \mathbf{x}}^{(q)} & e^{\mu} & & \mathbf{0} \\
& \ddots & \ddots & \ddots & \\
\mathbf{0} & & -e^{-\mu} & 2 m_{N-1, \mathbf{x}}^{(q)} & e^{\mu} \\
& & & -e^{-\mu} & 2 m_{N, \mathbf{x}}^{(q)}
\end{array}\right|
$$

In equilibrium $\left(\delta \sigma_{n}(\mathbf{x}) \rightarrow 0, \sigma_{n}(\mathbf{x}) \rightarrow \overline{\boldsymbol{\sigma}}\right)$, recursion relation $B_{n}=2 m^{(q)} B_{n-1}+B_{n-2}$ leads to the equilibrium value of $Y$ and $B$ as,

$$
\begin{aligned}
\bar{Y} & =Y[\boldsymbol{\sigma} \rightarrow \overline{\boldsymbol{\sigma}}]=e^{E / T} \\
\bar{B}_{n} \equiv B_{k, \cdots, k+n}(\boldsymbol{\sigma} \rightarrow \overline{\boldsymbol{\sigma}}) & = \begin{cases}\cosh [(n+1) E] / \cosh E & (n=\text { even }) \\
\sinh [(n+1) E] / \cosh E & (n=\text { odd })\end{cases}
\end{aligned}
$$

where $E=\sinh ^{-1}\left(m_{0}+\bar{\sigma}\right)$ denotes the one dimensional quark energy. By substituting $\bar{Y}$ in $R_{\mathbf{x}}$, we get the effective potential from the effective action (Eq. (2.6)),

$$
\mathscr{F}_{\text {eff }}(\overline{\boldsymbol{\sigma}})=S_{\text {eff }}(\overline{\boldsymbol{\sigma}}) / \sum_{x}=\frac{1}{2} \frac{2 N_{c}}{d} \overline{\boldsymbol{\sigma}}^{2}-T \log \left[\frac{\sinh \left[\left(N_{c}+1\right) E(\overline{\boldsymbol{\sigma}}) / T\right]}{\sinh [E(\overline{\boldsymbol{\sigma}}) / T]}+2 \cosh \frac{N_{c} \mu}{T}\right] .
$$

This has been obtained also in Ref. [4] and included in the effective potential in Ref. [7]. The critical values $T_{c}(\mu=0), \mu_{c}(T=0)$ and the tri-critical point $T_{\text {tcp }}$ can be extracted from the effective potential Eq. (3.6) and have been studied in Ref. [4] in the chiral limit,

$$
\begin{aligned}
T_{c}(\mu=0) & =d\left(N_{c}+1\right)\left(N_{c}+2\right) /\left[6\left(N_{c}+3\right)\right], \quad \mu_{c}(T=0) \simeq 0.55 \\
T_{\mathrm{tcp}} & =\left[\sqrt{225 N_{c}^{2}+20 d^{2}\left(3 N_{c}^{2}+6 N_{c}-4\right)}-15 N_{c}\right] /(20 d) .
\end{aligned}
$$


Finally, it is necessary to evaluate $R_{m n}^{(2)}$ to obtain the meson mass spectrum(See Eq. (2.10)). Here, the translational invariance simplifies the calculation. Since the system has translational invariance, $R_{\mathbf{x}}$ and $X_{N}$ are cyclic invariant for the temporal indices. By using this translational invariance we obtain,

$$
\begin{aligned}
&\left.\frac{\partial X_{N}}{\partial \sigma_{n}}\right|_{\bar{\sigma}}=\left.\frac{\partial X_{N}}{\partial \sigma_{N}}\right|_{\bar{\sigma}}=2 \bar{B}_{N-1} \\
&\left.\frac{\partial^{2} X_{N}}{\partial \sigma_{m} \partial \sigma_{n}}\right|_{\bar{\sigma}}=\left.\frac{\partial^{2} X_{N}}{\partial \sigma_{n^{\prime}} \partial \sigma_{N}}\right|_{\bar{\sigma}}=4 \bar{B}_{n^{\prime}-1} \bar{B}_{N-n^{\prime}-1} \quad\left(n^{\prime}=N-|n-m|\right) .
\end{aligned}
$$

It is found that Eqs. (3.2), (3.9) and (3.10) lead to,

$$
\left.R_{m n}^{(2)}\right|_{\bar{\sigma}}=\left.4\left(\bar{B}_{N-1}\right)^{2} \frac{\partial^{2} R}{\partial X_{N}^{2}}\right|_{\bar{\sigma}}+\left.4 \bar{B}_{n^{\prime}-1} \bar{B}_{N-n^{\prime}-1} \frac{\partial R}{\partial X_{N}}\right|_{\bar{\sigma}},
$$

in a straightforward calculation. In equilibrium, we can evaluate $\left.\left(d R / d X_{N}\right)\right|_{\bar{\sigma}}$ in the following way,

$$
\left.\frac{1}{\bar{R}} \frac{d R}{d X_{N}}\right|_{\bar{\sigma}}=\left.\frac{1}{\bar{R}} \frac{\partial R}{\partial \sigma_{n}}\left[\frac{\partial X_{N}}{\partial \sigma_{n}}\right]^{-1}\right|_{\bar{\sigma}}=\bar{\sigma} \frac{2 N_{c}}{d} \frac{1}{2 \bar{B}_{N-1}}
$$

where we used Eq. (2.9) and (3.4) in the final equality. Taking into account the even number of temporal lattice cite $(N=$ even), and substituting Eq. (3.5) and (3.12) into (3.11), we obtain,

$$
\frac{\bar{R}_{m n}^{(2)}}{\bar{R}}=-\bar{\sigma} \frac{2 N_{c}}{d} \frac{e^{i \pi m^{\prime}} \cosh \left[\left(N-2 m^{\prime}\right) E\right]}{\cosh E \sinh [E / T]}+\frac{\Delta \mathscr{R}}{\bar{R}} \quad\left(m^{\prime}=|n-m|\right),
$$

where $\Delta \mathscr{R}$ does not include temporal indices, and here we do not bother to write its explicit form.

\section{Meson mass}

Now we can explicitly evaluate the second variation of the effective action (Eq. (2.10)). Substituting Eq. (3.13) into (2.10), we obtain,

$$
\left.\delta_{\sigma}^{2} S_{\mathrm{eff}}\right|_{\bar{\sigma}}=\sum_{m n, \mathbf{x y}} \delta \sigma_{m \mathbf{x}}\left[V_{M}^{-1}(\mathbf{x y}) \delta_{m n}+\delta_{\mathbf{x y}} \overline{\bar{\sigma}} \frac{2 N_{c}}{d} \frac{e^{i \pi m^{\prime}} \cosh \left[\left(N-2 m^{\prime}\right) E\right]}{\cosh E \sinh [E / T]}\right] \delta \sigma_{n \mathbf{y}}
$$

where we ignore the effect of $\Delta \mathscr{R} / \bar{R}$ by requiring the null average condition: $\sum_{n} \delta \sigma_{n}=0$. We perform the Fourier transformation of Eq. (4.1) by using the translational invariance, $\sum_{n=1}^{N} \equiv$ $\sum_{m^{\prime}=1-m}^{N-m} \rightarrow \sum_{m^{\prime}=0}^{N-1}$ for the second term in Eq. (4.1), and we obtain,

$$
\left.\delta_{\sigma}^{2} S_{\mathrm{eff}}\right|_{\bar{\sigma}}=\sum_{\omega, \mathbf{k}} \delta \sigma_{\omega}(\mathbf{k})\left[\frac{2 N_{c} / d}{\sum_{j} \cos k_{j}}+\frac{\left(2 N_{c} / d\right) \overline{\boldsymbol{\sigma}}\left(\overline{\boldsymbol{\sigma}}+m_{0}\right)}{\cos \omega+2\left(\overline{\boldsymbol{\sigma}}+m_{0}\right)^{2}+1}\right] \delta \sigma_{\omega}(\mathbf{k}) .
$$

Here we introduce the prescription proposed in Ref. [9],

$$
(\omega, \mathbf{k})=(i M, \mathbf{0})+\left(\boldsymbol{\delta}_{v}\right) \pi,
$$



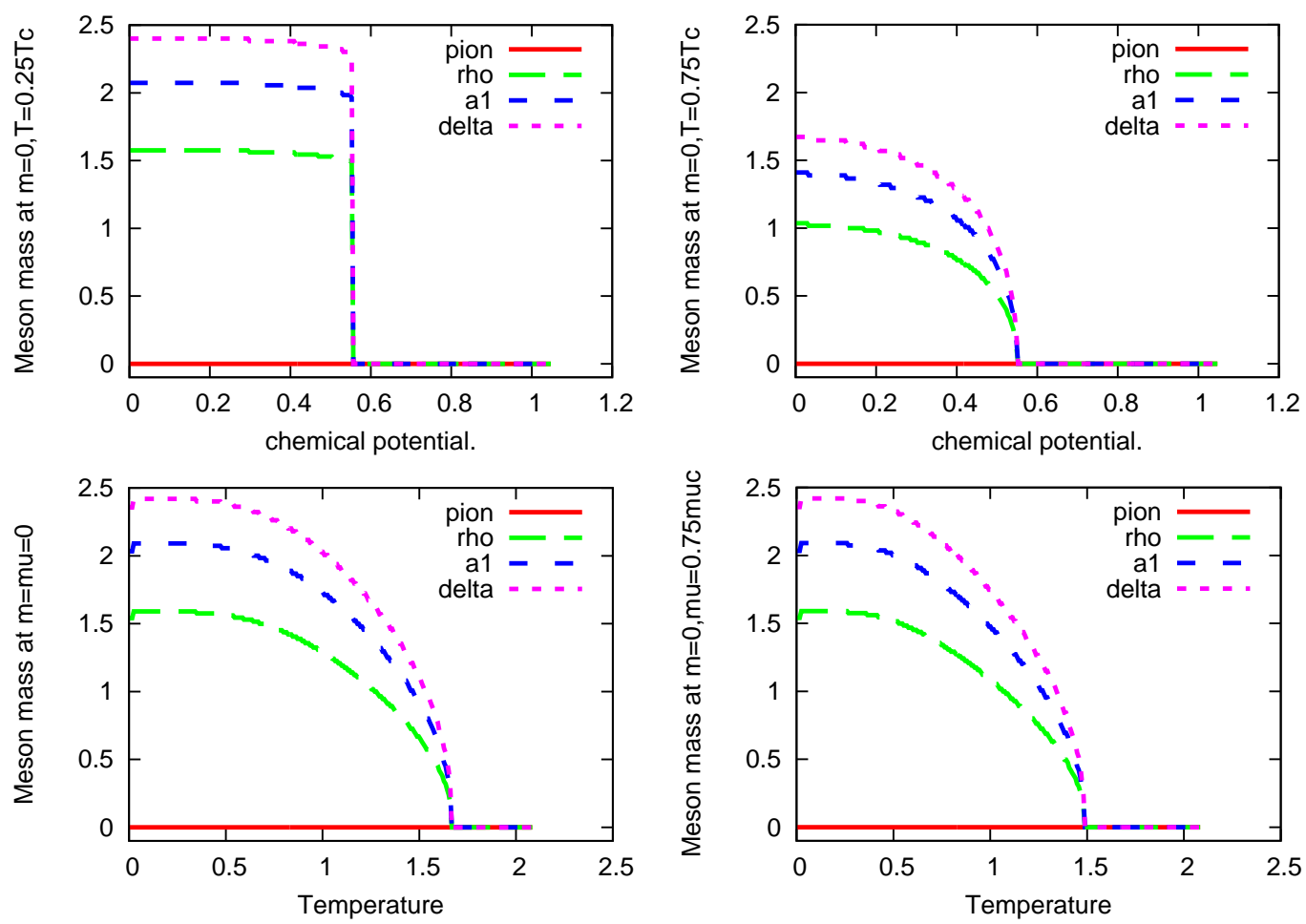

Figure 1: The $\mu$ dependence of meson mass at $T=0.25 T_{c}(\mu=0)$ (upper-left) and $T=0.75 T_{c}(\mu=$ 0 ) (upper-right) in the chiral limit. The $T$ dependence of meson mass at $\mu=0$ (lower-left) and $\mu=$ $0.75 \mu_{c}(T=0)$ (lower-right) in the chiral limit. Graphs are plotted in the lattice unit.

where $\left(\delta_{v}\right)$ is a " $d+1$ " dimensional vector which takes 0 or 1 and originates from the tastes degrees of freedom. In this prescription, " $M$ " is regarded as a meson mass. By putting the obtained inverse propagator for $\delta \sigma_{\omega}(\mathbf{k})$ equal to zero, the meson masses are found to be,

$$
\begin{aligned}
\pm \cosh M_{\kappa}(\bar{\sigma} ; T, \mu) & =2\left(\bar{\sigma}+m_{0}\right)\left(\frac{d+\kappa}{d} \bar{\sigma}+m_{0}\right)+1 \\
\kappa & \equiv \sum_{j=1}^{d} \cos \delta_{j} \in\{-d,-d+2, \cdots, d-2, d\} .
\end{aligned}
$$

Here, we tentatively take the plus sign to obtain the real number meson mass, and regard taste effects $\kappa$ as meson species in the same way as in Ref. [9]. In this scheme with $d=3$, " $\mathrm{\kappa}=$ $-3,-1,1,3$ " corresponds to " $\pi, \rho, a_{1}$ " and " $\delta$ " mesons, respectively.

For a small current quark mass $m_{0} \sim 0$, we obtain, $M_{\pi} \simeq 2 \sqrt{\bar{\sigma} m_{0}}$, which may be regarded as the PCAC relation. When the temperature $T$ and quark chemical potential $\mu$ are given, the chiral condensate $\bar{\sigma}(T, \mu)$ is obtained from the effective potential Eq. (3.6), then the meson mass $M_{\kappa}(\bar{\sigma} ; T, \mu)$ is determined by Eq. (4.4). In Fig. 1, we show $T$ and $\mu$ dependence of meson masses in the lattice unit. With a given $T$ lower than the tri-critical point $T_{\text {tcp }}$ (c.f. Eq. (3.8)), the meson masses except for pion discontinuously drop when $\mu$ passes through the critical value $\mu_{c}$, where the phase transition is first order (upper-left panel of Fig. 1). At higher temperatures, $T_{\text {tcp }} \leq T<T_{c}$, the phase transition is second order and thus the meson masses except for pion quickly but smoothly 
decrease to zero when $\mu$ approaches to $\mu_{c}$ (upper-right panel of Fig. 1). When $T$ approaches to its critical value $T_{c}$ with a given $\mu$ lower than $\mu_{c}$, the meson masses quickly and smoothly decrease to zero, where the phase transition is second order (lower panels of Fig. 1). The $T$ dependence of meson masses is slightly affected by a given $\mu$ in the chiral broken phase (lower panels of Fig. 1).

\section{Summary}

We have investigated the meson mass spectrum at finite temperature and density by considering the leading order of the $1 / d$ expansion in the strong coupling limit of lattice QCD. We have derived an analytical expression of meson masses as functions of the chiral condensate which is a function of temperature and chemical potential. We have thus explicitly studied the temperature and chemical potential dependence of meson masses near the critical value. We have confirmed that the pion mass satisfies the PCAC relation, and that masses of other mesons decrease to zero very quickly when the chemical potential or temperature approaches to the critical value.

\section{Acknowledgements}

This work is supported in part by the Ministry of Education, Science, Sports and Culture, Grant-in-Aid for Scientific Research under the grant numbers, 13135201, 15540243, 1707005, and 19540252.

\section{References}

[1] P. de Forcrand and S. Kim, Phys. Lett. B 645 (2007) 339 [hep-lat/ 0608012 ]; I. M. Barbour, S. E. Morrison, E. G. Klepfish, J. B. Kogut and M. P. Lombardo, Phys. Rev. D 56 (1997) 7063 [hep-lat/9705038].

[2] P. H. Damgaard, N. Kawamoto and K. Shigemoto, Phys. Rev. Lett. 53 (1984) 2211; Nucl. Phys. B 264 (1986) 1.

[3] G. Faldt and B. Petersson, Nucl. Phys. B 265 (1986) 197.

[4] Y. Nishida, Phys. Rev. D 69 (2004) 094501 [hep-ph/0312371].

[5] F. Karsch and K. H. Mutter, Nucl. Phys. B 313 (1989) 541; N. Bilic, F. Karsch and K. Redlich, Phys. Rev. D 45 (1992) 3228; V. Azcoiti, G. Di Carlo, A. Galante and V. Laliena, JHEP 0309 (2003) 014 [hep-lat/ 0307019 ]; Y. Nishida, K. Fukushima and T. Hatsuda, Phys. Rept. 398 (2004) 281 [hep-ph/0306066].

[6] P. H. Damgaard, D. Hochberg and N. Kawamoto, Phys. Lett. B 158 (1985) 239.

[7] N. Kawamoto, K. Miura, A. Ohnishi and T. Ohnuma, Phys. Rev. D 75 (2007) 014502 [hep-lat/0512023]; A. Ohnishi, N. Kawamoto, K. Miura, K. Tsubakihara and H. Maekawa, arXiv: 0704.2823 [nucl-th] ; A. Ohnishi, N. Kawamoto and K. Miura, J. Phys. G 34 (2007) S655 [hep-lat/0701024].

[8] N. Kawamoto and J. Smit, Nucl. Phys. B 192 (1981) 100; J. Hoek, N. Kawamoto and J. Smit, Nucl. Phys. B 199 (1982) 495; N. Kawamoto and K. Shigemoto, Nucl. Phys. B 237 (1984) 128; O. Martin, Phys. Lett. B 130 (1983) 411.

[9] H. Kluberg-Stern et al. Nucl. Phys. B 215 (1983) 527.

[10] P. Hasenfratz and F. Karsch, Phys. Lett. B 125 (1983) 308. 